\title{
HEAVY METAL OVERLOAD IN AUTISTIC CHILDREN
}

\author{
By \\ El Safty A.M.K \\ * Department of Occupational and Environmental Medicine, \\ Faculty of Medicine, Cairo University .
}

\begin{abstract}
:
Background: An enormous, alarming, and unexplained increase in the prevalence of autism is being reported, on an almost daily basis everywhere. Exposure to certain neurodevelopmental toxins, may increase the risk of autism spectrum disorders (ASD), autism, and attention-deficit hyperactivity disorder. Heavy metals including lead, aluminum and ethylmercury interrupt growth factor signaling, delaying neurodevelopmental growth of infants and children. Aim of the work: studying the state of some heavy metal overload and trace elements among children with autistic features. Subjects and Methodology: children diagnosed as having autistic features were recruited from pediatric psychiatric clinic at Kasr Al Aini and National Research center. A total of 64 cases and 30 matched children as referent group, were involved in our cross sectional study. Measuring levels of heavy metals including RBCs lead, urinary mercury, serum aluminum, and zinc was performed using the atomic absorption spectrophotometer. Results and discussion: Abnormal high levels of lead, mercury and aluminum, low level of zinc, were detected in the autistic featured group. The measured levels of heavy metals and trace elements were statistically significantly different when compare with that in referent group. Conclusion and recommendation: The level of heavy metals should be monitored in every case diagnosed as having autistic features. We recommend early intervention for preventing long term disability. A full management protocol including early diagnosis, behavioral, speech therapy, chelation and replacement therapy should be developed.
\end{abstract}




\section{Introduction}

Autism and autistic like disease can be caused by a number of disorders, including Fragile X Syndrome, tuberous sclerosis, and phenyl-ketonuria and by at least one notable chromosomal abnormality, an inverted duplication of a portion of chromosome 15. But for the vast majority of cases of autism today, there is no strictly genetic explanation. There is general agreement that most cases of autism arise "from the interaction of an early environmental insult and a genetic predisposition" (Trottier et al, 1999; Bristol et al, 1996).

Several recently-released reports point to the occurrence of an autism "epidemic" with the latest incidence figures quoted to be on the order of 1 out of every 250 children. The Report on Autism to the California Legislature (Diagnostic and Statistical Manual of Mental disorders (DSM IV) released in 1999 documents a large increase in full-blown autism alone, with other disorders increasing at the same rate as population growth (Spitzer and Wakefield.,1999),(Mezzich .,2002). A more recent study by Herbert at 2010, suggested a substantial increase in ASD prevalence of one in 150 that was explained by altered gene variants in ASD due to environmental stressor and exposures.
Yazbak" 2005' found similar rates of increasing incidence in other states reported in his "Autism 99: A National Emergency". The Center for Disease Control's own investigation of Brick town-ship, New Jersey found a very high incidence of autism as well. Some noted sources attribute the apparent increase in autism incidence to better diagnosis on the part of pediatricians and the various pediatric specialties. Most, however, are unable to fully accept this simplistic explanation because the diagnosis is strictly a behavioral one, and it is highly doubtful that the highly skilled diagnosticians of earlier years could have overlooked such obvious behavioral anomalies occurring in such a large proportion of children. Furthermore, since it is impossible to have a "genetic epidemic", one must examine possible early environmental insults for clues to explain the increase in autism cases..

The researchers found that exposure to toxins such as ethanol and heavy metals (including lead, aluminum and the ethylmercury-containing preservative thimerosal) interrupt growth factor signaling. This adversely impacts methylation reactions such as the transfer of carbon atoms. Methylation is critical to proper neurological development in infants 
and children. Now, most vaccines in the United States and Europe contain only trace amounts of thimerosal. However, multidose flu vaccines still contain thimerosal. The same is true of many larger, multidose vials of vaccines shipped to and used in developing countries. http://www. medicineonline.com/conditions/article. html?articleID=721

Individuals diagnosed as Autism Spectrum Disorders "ASD" have in common, delayed or disabilities in social skills such as ordinary conversation, eye contact and emotional understanding of others. They also share delays in fine and gross motor skills. These issues are likely to lead to impaired social interaction, problems with verbal and nonverbal communication, and unusual, repetitive, or severely limited activities and interests. It's important to note, that "symptoms" of autism can also be strengths. For example, a child on the autism spectrum may have better observational abilities than a typical child of the same age. Another commonly available test is blood or urine pyruvic acid. Pyruvic acid can be elevated for a number of reasons, but mercury is notorious for interfering with the mitochondrial pyruvate dehydrogenase complex, where it binds to and deactivates the lipoic acid coenzyme, resulting in elevated pyruvic acid. Mercury and other heavy metals interfere with heme synthesis, leading to urinary excretion of uroporphyrin and coproporphyrin. Mercury also causes production of precoproporphyrin, which may be considered a specific marker for mercury poisoning (Aman and Langworthy, 2000).

Most experts agree that early intervention is a key to treating autism. While there is no sure cure for the disorder, there are a wide range of treatments that can help reduce symptoms, teach skills, and generally improve individuals' prognoses (Herbert., 2010).

Most autistic children suffer from some degree of intestinal dysbiosis, abnormal intestinal permeability and nutritional derangements (Adams and Conn,1997), which must all be corrected as much as possible prior to any attempt at detoxification. Many of the drugs and supplements used for mercury detoxification are rich sources of nutrition for bacteria and fungi (Bryson, 1997).

There is new evidence that autism can be caused by a biochemical abnormality, which disables metallothionin (MT) protein accompanied by early exposure to neurotoxins such as mercury. Autistic children exhibit evidence of oxidative stress and impaired methylation, which may reflect effects of toxic exposure on sulfur metabolism (Deth et al., 2008). 
Mechanisms with the potential for disrupting MT functioning include severe $\mathrm{Zn}$ depletion, impaired synthesis of Glutathione (GSH), toxic metal overload, a pyrrole disorder, and a sulfur amino acid abnormality, along with a myriad of other genetic and/or environmental insults. Other factors that have been considered in the aetiology of ASD include: maternally derived antibodies, maternal infection, folic acid supplementation, epigenetics, measles, mumps, rubella vaccination, and even electromagnetic radiation. In each case, the consequences, whether direct or indirect, negatively affect the nervous system, neurodevelopment, and environmental responsive genes (Currenti ., 2009).

Blaylock and Strunecka

(2009) brought evidence that most heterogeneous symptoms of ASD have a common set of events closely connected with dysregulation of glutamatergic neurotransmission in the brain with enhancement of excitatory receptor function by pro-inflammatory immune cytokines as the underlying mechanism. They also suggested that environmental and dietary excitotoxins, mercury, fluoride, and aluminum can exacerbate the pathological and clinical problems by worsening excitotoxicity and by microglial priming. In addition, each has effects on cell signaling that can affect neurodevelopment and neuronal function.
Depletion or deficiency of the cellular antioxidant systems is seen in a number of autistic children. A common finding in autistic children is an abnormally low erythrocyte glutathione level. Low glutathione levels in the red cells are not specific for heavy metal toxicity, but may be supporting evidence. The potential causes for this deficiency in cellular antioxidant substances are congenital or exposure to toxins. Heavy metals are well-documented causes of intracellular antioxidant depletion. The discovery of disordered metal-metabolism in autism may lead to early identification of autismprone children, prevention of regressive autism, and improved therapy outcomes (Adams and Conn, 1997). Whether the cause is too little production of glutathione, rapid consumption or a combination of both, many of these children can benefit from exogenous antioxidant support. Nacetylecycteine (NAC) and meso-2,3dimercaptosuccinic acid (DMSA) was investigated by Gürer and his colleagues in 1998 to study its capability of reversing parameters indicative of lead-induced oxidative stress. They concluded that treatment with either (NAC) or (DMSA) reversed lead-induced alterations in MDA and GSH RBC's content, providing evidence of the reversibility of oxidative stress by treatment with a thiol antioxidant (NAC), as well as a chelating agent DMSA. 


\section{Aim of the work}

To study the state of some heavy metal overload and trace elements among children with autistic features.

\section{Methodology}

In response to the increasing number of children referred for evaluation of the level of heavy metal overload in children with autistic features, our study was designed as cross-sectional one to investigate the level of some of the heavy metals and trace elements among the children diagnosed as having ASD. Children referred to investigation from the outpatient clinic for developmental disorders belonging to the psychiatric department at Kasr Al Ainy Hospital. This is the largest hospital at the middle east serving patients recruited from all over Egypt. Children were further assessed by psychiatric physicians attending the outpatient clinic by fulfilling a questionnaire and clinical examination. Children were complaining mainly from delayed language development and attention deficit. Parental consent and approval from the Department Ethical Committee was taken prior to children involvement in the study. The total duration of the study was 9 months starting from January 2009 till September 2009. All attendant children to the clinic were involved (number 86), however only 64 accepted joining this research. A referent group of 30 children was randomly selected from a nearby preschool nursery.

After fulfilling the clinical examination and audiometric testing to exclude auditory defects, the following investigations were performed: RBC's lead, serum aluminum, urinary mercury, and serum zinc.

\section{Results and Discussion}

Table (1) General characteristics of the studied group:

\begin{tabular}{|c|c|c|c|c|c|c|}
\hline General characteristics & \multicolumn{2}{|c|}{ Cases } & \multicolumn{2}{|c|}{ Referent group } & \multicolumn{2}{|c|}{ Test of sig. \& P value } \\
\hline \multirow{2}{*}{ Age (yrs.) } & Mean & S.D & Mean & & \multirow{2}{*}{0.2167} & \multirow{2}{*}{0.479} \\
\hline & 3.153 & 1.283 & 3.257 & 1.295 & & \\
\hline Sex & No. & $\%$ & No. & $\%$ & \multirow{3}{*}{0.115} & \multirow{3}{*}{0.73} \\
\hline Male & 49 & 76.6 & 22 & 73.3 & & \\
\hline Female & 15 & 23.4 & 8 & 26.7 & & \\
\hline \multirow{3}{*}{$\begin{array}{l}\text { Socioeconomic status: } \\
\text { Middle } \\
\text { Low }\end{array}$} & No. & $\%$ & No. & $\%$ & \multirow{3}{*}{0.103} & \multirow{3}{*}{0.74} \\
\hline & 30 & 43.8 & 13 & 43.3 & & \\
\hline & 34 & 53.2 & 17 & 56.7 & & \\
\hline
\end{tabular}


The recruited group age ranged from 2-6 years old $(2.7+0.8)$. Socioeconomic status (SES) that is typically categorized according to (income, education, and occupation of parents) was assessed. Our group was in the middle $(43.8 \%)$ and low $(53.2 \%)$ SES. Both the recruited groups (cases and referent groups) showed no statistically significantly difference as regards age, sex and socioeconomic status.
It is clear from our work that the prevalence of ASD shows no predilection to specific socioeconomic state, but is more likely to occur in boys $76.6 \%$ than girls $23.4 \%$, meaning more than 3 folds. Similarly, Newschaffer et al., 2007 studied the epidemiology of ASD and concluded that the averages male to female ratio is $4.3: 1$ male-to-female.

Table (2) Clinical findings encountered in the cases with autistic features

\begin{tabular}{|l|cc|}
\hline \multicolumn{1}{|c|}{ Clinical manifestations (No. 64) } & Number & $\%$ \\
\hline Communication & 53 & 82.8 \\
Avoid eye contact & 22 & 34.4 \\
Seem deaf & 36 & 56.3 \\
language regressive skills & & \\
Social relationships & 5 & 7.8 \\
Appear unaware of others & 4 & 6.3 \\
Attack others without provocation & 24 & 37.5 \\
Exploration of environment & 34 & 53.1 \\
Fixate on single item or activity & 9 & 14.1 \\
Practice strange actions like rocking or hand-flapping \\
(repetitive, stereotyped activities)
\end{tabular}


Full history and clinical examination of the studied groups showed that non of the referent group suffered from any behavioural disabilities. Almost eighty three percent of the cases with autistic features shows lack of eye contact, while only $34 \%$ seems to be deaf "however their audiometric results denies that), history of language regressive skills (start developing language, then abruptly stop talking) was described in $56 \%$ of the sample. Similar results were documented by Tuchman and Rapin (1997) demonstrated that thirty percent of their studied autistic children were suffering from regression of language, sociability and behavior. Concerning the behavioral manifestation encountered in our study, only $8 \%$ of the sample experiences unawareness of others while $6 \%$ shows aggression towards others. Other psycho-behavioral diagnostic characteristic of autism is the presence of repetitive, stereotyped activities and the need for sameness that is manifested in 24 cases of our studied cases. As for the history of epileptic attacks, only 3 cases experience those attacks. Comparably, the prevalence of epilepsy in children with ASD was estimated to range from $7-42 \%$ (Tuchman, 2000). Similarly, Manning-Courtney et al, 2003 described children with ASD are characterized by deficits in the three core areas of communication, reciprocal social interaction and the presence of stereotyped and repetitive behaviors.

Table (3) Mean levels of measured parameters among the studied groups.

\begin{tabular}{|c|c|c|c|c|}
\hline \multirow[t]{2}{*}{$\begin{array}{l}\text { Measured } \\
\text { parameters }\end{array}$} & \multirow[t]{2}{*}{$\begin{array}{l}\text { Normal } \\
\text { Values }\end{array}$} & $\begin{array}{c}\text { Autistic } \\
\text { featured } \\
\text { children group }\end{array}$ & Referent group & $\begin{array}{c}\text { Test of sig. \& } \mathbf{P} \\
\text { value }\end{array}$ \\
\hline & & Mean & Mean & \\
\hline RBCs Lead & $<10 \mathrm{Ug} / \mathrm{dL}$ & $23.90+10.78$ & $8.50+3.77$ & $4.2589<0.001$ \\
\hline Urinary Mercury & 4-10 Ug/L & $6.14+$ & $3.58+1.14$ & $3.0175<0.005$ \\
\hline Serum Aluminum & $<0.6 \mathrm{Ug} / \mathrm{dL}$ & $0.43+0.049$ & $0.346+0.036$ & $<0.01$ \\
\hline Serum Zinc & $70-130 \mathrm{Ug} / \mathrm{dL}$ & $74.60+15.52$ & $98.60+10.99$ & $<0.01$ \\
\hline
\end{tabular}


Table (4) Prevalence of abnormal levels of heavy metals and trace elements in the studied group.

\begin{tabular}{|l|c|c|}
\hline & Number of Cases & $\%$ \\
\hline High RBC>s Lead & 49 & 76.6 \\
\hline High urinary Mercury & 5 & 7.8 \\
\hline High Serum Aluminum & 12 & 18.8 \\
\hline Low Serum Zinc & 36 & 56.3 \\
\hline
\end{tabular}

Table (3) \& (4) shows the level of the measured heavy metals in our studied groups. There was statistically significantly higher lead, mercury and aluminum in autistic children group compared to the referent group. However lower serum zinc levels were demonstrated in the selected cases compared to the referent group. The RBCs lead levels were higher than normal values in $76.6 \%$ of cases. In-spite of normal mean level of urinary mercury in the studied groups, yet abnormally high levels were found in 5 cases (7.8\%). High serum aluminum levels were found in 12 cases $(18.8 \%)$. Zinc deficiency was manifested in $56.3 \%$ of the studied group explaining the exacerbation of the clinical presentation of most of the cases. Zinc usually shows inverse association with high lead levels.
Zinc depletion may act as metallothionin disruptors impairing synthesis of GSH and toxic metal overload (Dethet al., 2008).

The most likely explanation for the severe metal-metabolism dysfunction lies in the function of a group of proteins known as metallothioneins. Genetic errors in the genes coding for the various proteins or having the genes "turned off" by influences on the promoter regions on either side of the genes themselves would be the only two explanations for dysfunction of this group of proteins. The production of metallothionein proteins has possibly turned off by environmental factors. Currenti in 2010 studied the effect of multiple environmental exposures on the mutations resulting in wide phenotypic spectrum known as ASD. 
The use of medication in children with autism is evolving, with new studies aiding practitioners in ongoing medical management. Medication should never be the first line of treatment. Medications are used to target specific symptoms associated with ASD and are always considered "adjuvant" treatment in combination with other treatments and interventions.

\section{Recommendations}

Because studies are limited in the use of medication in children with ASD, practitioners are encouraged to share their clinical experiences in the use of these medications. Controlled study trials are needed to examine effects of medications and to measure outcomes. Outcome measures need further refinement in order to provide accurate appraisals of these treatments. As more of these outcomes are shared, protocols can be devised to guide clinicians in the treatment process. As we build the knowledge base of autism treatment, the hope is that we expand treatment options and improve quality of life for those children.

We recommend the development of a multidisciplinary team made up of a psychologist, a neurologist, a psychiatrist, a speech therapist, behavioural therapist and toxicologist to handle safely the diagnosis and treatment children with ASD.

\section{References}

1. Adams L., and Conn S.(1997): Nutrition and its relationship to autism. Focus on Autism \& Other Developmental Disabilities.12, 53-58.

2. Aman M.G. and Langworthy K. S. (2000): Pharmacotherapy for hyperactivity in children with autism and other pervasive developmental disorders. Journal of Autism and Developmental Disorders. 30, 451-459.

3. Bryson S.(1997): Epidemiology of autism: Overview and issues outstanding. In D. J.Cohen and F. R. Volkmar (Eds.), Handbook of autism and pervasive developmental disorders(2nd ed., pp. 41-46). New York: Wiley.

4. Blaylock R.L. and Strunecka A.(2009): Immune-glutamatergic dysfunction as a central mechanism of the autism spectrum disorders.Curr Med Chem. 16(2):157-70. http://www.generationrescue.org/blaylock/ Immunoexcitotoxicity.pdf

5. Bristol D.W., Wachsman J.T. and Greenwell A. (1996): The NIEHSPredictive-Toxicology Evaluation Project. Environ. Health Perspect. 104 (5): 1001-1010.

6. Currenti S.A. (2009): Understanding and Determining the Etiology of Autism.Cell MolNeurobiol. Sep 23. http://www.springerlink. com/content/e1120810226n65t7/

7. Currenti S.A. (2010): Understanding and determining the aetiology of autism. Cell Mol. neurobio. 30:161-171.

8. Deth R., Muratore C., Benzecry J., PowerCharnitsky V.A and Waly M. (2008): How environmental and genetic factors combine to cause autism: A redox/methylation. Hypothesis Neurotoxicology. 29(1):190-201.

9. Gürer H., Ozgünes H., Neal R., Spitz D.R. and Erçal N. (1998): Antioxidant effects of $\mathrm{N}$-acetylcysteine and succimer in red blood cells from lead-exposed rats. Toxicology. 17;128(3):181-9. 
10. Herbert M.R. (2010): Contributions of the environment and environmentally vulnerable physiology to autism spectrum disorders. Curr. Opin. Neurol. 23(2):103-10. http://www.ncbi. nlm.nih.gov/pubmed/20087183.

11. Manning-Courtney P., Brown J., Molloy C., Reinhold J., Murray M. and Sorensen-Burnworth R, (2003): Diagnosis and treatment of autism spectrumdisorders. Current Problems in Pediatric and Adolescent Health Care.33:283-304.

12. Mezzich J.E. (2002): "International Surveys on the Use of ICD-10 and Related Diagnostic Systems". Psychopathology 35 (2-3): 7275. doi:10.1159/000065122. PMID 12145487. http://content.karger.com/ProdukteDB/ produkte.asp?Aktion $=$ Show Abstract $\&$ Arti kelNr $=65122 \&$ Ausgabe $=228600 \&$ Produkt $\mathrm{Nr}=224276$. Retrieved. 2008-09-02.

13. Newschaffer J. C., Croen A.L., Daniels J., Giarelli. E. Grether J.K., Levy S.E., Mandell D.S., Miller L.A., Pinto-Martin J., Reaven J., Reynolds A.M., Rice C.E., Schendel D., and Windham G.C. (2007): The Epidemiology of Autism Spectrum Disorders. Annu. Rev. Public Health. 28:21(1):21.24.
14. Spitzer RL and Wakefield JC. (1999): DSM-IV diagnostic criterion for clinical significance: does it help solve the false positives problem? Am J Psychiatry. 156(12):1856-64. PMID 10588397

15. Trottier G., Srivastava L.,Walker C. D.(1999): Etiology of infantile autism: A review of recent advancements in genetic and neurobiological research. Journal of Psychiatry \& Neuroscience,24, 103-115.

16. Tuchman R.F., and Rabin L. (1997): Regression in pervasive developmental disorders, seizures and epileptiform electroencephalogram correlates. Pediatrics, 99, 560-565,

17. Tuchman L.(2000): Treatment of seizure disorders and EEG abnormalities in children wirh autistic spectrum disorders. Journal of autism and developmental disorders. 30, 485489.

18. Yazbak, F.E.(2004): "Autism seems to be increasing worldwide, if not in London" - Letter

- British Medical Journal; 328:226-227. http:// wWw.medicineonline.com/conditions/article. html?articleID=721. 